\title{
Transtorno de Estresse Pós-Traumático (Tept) e Coping em Trabalhadores do Setor Aeroespacial Brasileiro: O Caso de Alcântara, MA
}

\author{
Post Traumatic Stress Disorder (Ptsd) and the Relationship Between Coping in \\ Aerospace Workers: The Alcantara'S Case \\ Trastorno de Estrés Postraumático y Coping en Trabajadores del Sector Aeroespacial \\ Brasileno: El Caso de Alcântara, MA \\ Liliana Andolpho Magalhães Guimarães ${ }^{I}$ \\ Universidade Católica Dom Bosco (UCDB) \\ Sérgio Nolasco Hora das Neves \\ Universidade Estadual de Campinas (UNICAMP)
}

\begin{abstract}
Resumo
Esse estudo caracterizou, antes e depois do tratamento psicofarmacológico-psicoterápico, a frequência do Transtorno de Estresse Pós-Traumático-TEPT, sintomas depressivos e ansiosos em trabalhadores do setor aereoespacial, identificando o método de coping utilizado e relacionando-o à resposta ao tratamento. A 18 trabalhadores ligados à explosão do foguete VLS-1 aplicou-se Entrevista clínica psiquiátrico-psicológica, Questionário de Seqüelas do Trauma, Escala de Ansiedade de Hamilton, Inventário de Depressão de Beck e Escala de Coping de Billings e Moos. Dos 18 participantes, 10 (55,5\%) apresentaram TEPT, $6(33 \%)$ sintomas ansiosos e $8(44 \%)$ depressivos. Em $15(72.3 \%)$ o coping foi ativo e em 3 (27.7\%), passivo. Após 3 meses de tratamento, dos 10 participantes com TEPT, 8 (80\%) não o apresentavam mais e nem sintomas ansiosos, um manteve os sintomas depressivos. Os 2 $(20 \%)$ que não melhoraram tinham um coping passivo. A frequência de TEPT foi alta $(55,5 \%)$ e os coping ativo $(80 \%)$ tiveram rápida melhora sintomática.
\end{abstract}

Palavras-Chave: Transtorno de Estresse Pós-Traumático, trabalhadores, aeroespacial, coping, tratamento.

\begin{abstract}
This study aim to investigate the prevalence of Post Traumatic Stress Disorder-PTSD, anxiety and depressive symptoms, before and after combined psychopharmacological and psychological treatment in a group of aerospace workers, identifying the coping method used by the participants and its relation to response to treatment. Eighteen workers linked to VLS-1 rocket blaster, were assessed by means of clinical psiquiatric-psychological interview, STQ Questionnaire, HAM-A, BDI, and Billings \& Moos Coping Scale. The 10 (55.5\%) out of 18 participants presented with PTSD, with anxiety $6(33 \%)$ and depressive symptoms $8(44 \%) ; 15(72.3 \%)$ out of 18 had an active coping and $3(27.7 \%)$ a passive one. After three months of treatment, $8(80 \%)$ out of 10 participants with PTSD made a full recovery. The two subjects $(20 \%)$ who did not improve had a passive coping. PTSD frequency was high $(55.5 \%)$ and $80 \%$ of those with PTSD responded quickly to the treatment and had an active coping.

Key Words: Post Traumatic Stress Disorder; workers; aerospace; coping; treatment.
\end{abstract}

\section{Resumen}

Este estudio caracterizou antes y después del tratamiento psicofarmacológico-psicoterápico, la frecuencia del Trastorno de estrés postraumático (TEPT), sintomas depresivos y de ansiedade, en trabajadores del sector aeroespacial, identificando o método de coping utilizado, com a respuesta al tratamiento. A los 18 empleados de la explosión del cohete VLS-1 se aplicó: Entrevista clinica psiquiatrico-psicológica, Cuestionario sobre las secuelas de trauma, Escala de Ansiedad de Hamilton, Escala de Depresión de Beck y Escala de Coping de Billings y Moos. De los 18 participantes, $10(55 \%)$ tenian trastorno de estrés postraumático, sintomas de ansiedad $6(33 \%)$ y 8 (44\%) depresivos; 16 (72\%) tuvieran coping activo e 3 (27,7\%) pasivo. Después de tres meses de tratamiento, los 10 participantes com TEPT, 8 (44\%) no presentaran más sintomas, uno continuó con los sintomas depresivos e ninguno con sintomas de ansiedad. Los $2(20 \%)$ que no mejoraran tenian un coping pasivo. La frecuencia del TEPT fue alta $(55.5 \%)$ y los participantes con coping activo $(80 \%)$ mostraran una rápida mejoria de los sintomas. Palabras clave: Trastorno de estrés postraumático; trabajadores; sector aeroespacial; coping; tratamiento

\section{Introdução}

O interesse e o envolvimento do Brasil em atividades espaciais têm ocorrido desde os anos 1960, e os avanços tecnológicos obtidos são

1 Endereço: Rua Brás Cubas, 105 Jd Nossa Sra Auxiliadora 13075-500 Campinas, São Paulo, Brasil.Tel (019) 92980721 expressivos, apesar das dificuldades econômicosociais enfrentadas pelo setor. Em agosto de 2003, uma grande explosão destruiu o foguete VLS1 (Veiculo Lançador de Foguetes) no Centro de Lançamento de Alcântara, Maranhão, Brasil, durante os preparativos para seu lançamento, na denominada Operação São Luiz, matando 21 técnicos civis do Instituto de Aeronáutica e Espaço (IAE) do Centro 
Técnico Aeroespacial (CTA), São José dos Campos, São Paulo, Brasil. No país, este, foi o primeiro acidente com vitimas, no setor aeroespacial. Em outubro de 2003, uma equipe de Saúde Mental coordenada pela primeira autora e composta por psicólogos e psiquiatras iniciou um trabalho intensivo de diagnóstico e tratamento psicológico-psiquiátrico, encerrado em março de 2005. Este artigo aborda um dos sub-projetos realizados, "Transtorno de Estresse Pós-Traumático (TEPT) e coping em trabalhadores do setor aeroespacial brasileiro: o caso de Alcântara, MA".

\section{Breves considerações sobre o TEPT}

O TEPT tem por definição diagnóstica a ocorrência de um grave estresse traumático como fator etiológico desencadeante. Após a exposição, inicia-se uma série variável de sintomas característicos e perturbações de natureza psicológica, social e biológica.

Segundo a APA (2005) o TEPT é classificado nos Transtornos de Ansiedade (F43.1 - 309.81 Transtornos de Estresse Pós-Traumático) e caracteriza-se pelo surgimento de sintomas específicos após a exposição a um evento traumático, os quais compõem a seguinte tríade de dimensões psicopatológicas classificados por letras, sendo que pelo menos uma manifestação de cada agrupamento de sintomas deve estar presente após o evento traumático: (i) revivência persistente do evento através de recordações, imagens e pensamentos, sonhos e agir ou sentir como se estivesse ocorrendo novamente através de ilusões, flashbacks ou apenas por um sentimento vívido. Estas revivências são recorrentes e causam grande aflição; (ii) esforço persistente em evitar lembranças e pensamentos sobre o evento, situações em que o evento possa ocorrer novamente e coisas, pessoas, lugares e situações que não têm ligação direta com o evento, mas que de alguma forma ativam a sua lembrança; (iii) estado de ansiedade e excitabilidade constantes com manifestação de insônia, surtos de agressividade, dificuldade de concentração, irritabilidade e hipervigilância.

Os sintomas devem estar presentes por pelo menos há um mês. O TEPT é sub-classificado em agudo quando a duração é de um a três meses, crônico quando a duração é superior a três meses e de início tardio quando se iniciam seis meses após o evento traumático (APA, 2005).

Estudos de caso- controle ainda são raros no mundo e no Brasil. Um importante estudo epidemiológico foi realizado na cidade de São Paulo, envolvendo três estudo de caso-controle: (i) um neuropsológico, (ii) um de neuroimagem estrutural e (iii) outro de neuroimagem molecular, cada um focado em diferentes objeticos, fornecendo informações complementares (Bressan et al, 2009).

No Brasil, não existem dados epidemiológicos sobre o TEPT (Kapczinski, 2003), em particular o relacionado ao trabalho. Nos ambientes de trabalho especificamente, o TEPT causa sofrimento clinicamente significativo e/ou prejuízo no funcionamento ocupacional, repercutindo em outras áreas importantes da vida ( Glina, Rocha, Batista \& Mendonça, 2001). Os eventos traumáticos mais comumente encontrados são a violência ou ameaça de violência, e.g., acidentes e desastres graves com morte ou, morte repentina ou problema grave de saúde, assalto à mão armada, briga entre trabalhadores, entre outros (Braverman, 1998).

Existem estudos sobre TEPT em grupos ocupacionais específicos: policiais enfermeiros, entre outros, sendo que os trabalhadores mais estudados têm sido os bombeiros (Negir, Farci, Sampaio \& Guimarães, 2003).

Não se encontrou na pesquisa bibliográfica realizada para esse estudo, nenhuma referência nacional ou internacional que fornecesse dados comparativos sobre TEPT em trabalhadores com as mesmas características do grupo investigado.

O TEPT é um transtorno psiquiátrico freqüente, muitas vezes não diagnosticado, e com tendência a cronicidade, que se não tratado adequadamente, pode tornar-se altamente incapacitante e refratário às intervenções terapêuticas (Kessler, Sonnega, Bromet, Hughes \& Nelson, 1995).

\section{Tratamento}

Ao se tratar um paciente com TEPT deve-se ter em mente a complexidade do transtorno e a necessidade de um tratamento multidimensional. Existem três componentes importantes para o tratamento do após o evento traumático: i) a intervenção precoce (Bryant, 2005); ii) a reincorporação ao trabalho e iii) o acompanhamento (Yehuda, 2002).

$\mathrm{Na}$ pesquisa bibliográfica realizada para a viabilização desse estudo contatou-se a existência de poucos estudos controlados envolvendo intervenções psicoterápicas e/ou farmacológicas na prevenção secundária do TEPT. Cabe ressaltar que, um paciente pode necessitar de diferentes fármacos no decorrer do tratamento, a depender dos sintomas que forem mais proeminentes em determinado momento.

A abordagem terapêutica mais eficaz referida é obtida pela associação farmacoterápica e psicoterápica (Yehuda, 2002; Berlim, Perizzolo, Fleck, 2003; Amor, Sánches, Gancedo \& Gonzáles, 2008), (especialmente a cognitivo-comportamental) adotadas neste estudo, nos formatos individual e grupal.

Com base em ensaios abertos e randomizados é proposta a seguinte associação entre as dimensões psicopatológicas do TEPT e a resposta aos diferentes fármacos (Davidson, 1992): i) os sintomas de revivescência do trauma mostram melhor resposta aos antidepressivos tricíclicos e IMAOs; ii) a esquiva a estímulos relacionados ao trauma e distanciamento afetivo aos ISRS; iii) a hiperestimulação autonômica 
aos betabloqueadores e agonistas alfa-2-adrenérgicos.

Não existe até o momento um fármaco que seja igualmente eficaz na remissão das três dimensões psicopatológicas do TEPT, sendo utilizados antidepressivos, ansiolíticos, anticonvulsivantes, anti-hipertensivos e antipsicóticos, de acordo com a sintomatologia mais proeminente (Kapczinski, 2003).

Há um número expressivo de ensaios clínicos sugerindo que certas medicações, especialmente antidepressivos com propriedades serotoninérgicas, podem ser úteis no tratamento do TEPT (Kats et al, 1998-1995).

Novas abordagens terapêuticas, incluindo o uso de novos anticonvulsivantes estão em fase de testes (Kapzinski, 2003).

\section{Coping: Fator de risco ou de proteção ao TEPT}

A atenção aos fatores de risco e proteção ao TEPT tem se ampliado ( Katz et al, 1994-1995). São considerados fatores de risco: (i) a exposição a situações traumáticas prévias como: abuso, ser do sexo feminino (proporção 2:1 a prevalência mulheres-homens), (ii) história pessoal e familiar de outros transtornos mentais, (iii) separações precoces e eventos traumáticos na infância.

Também no âmbito ocupacional, algumas variáveis internas ou psicológicas individuais têm sido estudadas mais sistemática e freqüentemente, e podem atuar como preditoras da ocorrência do TEPT, contribuindo para seu prognóstico, tais como: estratégias de coping, resiliência, hardiness, locus de controle, entre outras.

Esta investigação centrou-se na relação da resposta ao tratamento ao TEPT com o coping, que pode ser definido como uma forma de enfrentamento que objetiva prevenir, evitar ou controlar o distress emocional, ou os constantes e mutáveis esforços cognitivos e comportamentais utilizados pelo indivíduo para lidar com demandas internas e/ou externas específicas, caracterizadas como estressores, que sobrecarregam ou excedem seus recursos pessoais (Lazarus, 1966).

O coping é determinado por fatores pessoais, exigências situacionais e recursos disponíveis (Rutter, 1988). Deve ser examinado em um contexto específo (Krum \& Bandeira, 2008) pois os pensamentos ou ações a ele relacionados, são sempre direcionados a condições particulares. Quanto mais definido e focal for o contexto, mais fácil será ligar um ato ou pensamento exclusivo de coping a uma demanda situacional. Além disso, é necessário considerar o coping como um processo de constante transformação, no qual as estratégias utilizadas em determinado momento, podem não ser as mesmas das avaliadas como adequadas ao enfrentamento de outra situação, que remetem à avaliação, a interpretação e a representação cognitiva do fenômeno percebido e a reavaliação desses em função das mudanças ocorridas na relação com o indivíduo (Rutter, 1988; Lazarus, 1966).

Alguns autores (Rutter, 1988; Billings \& Moos, 1981) consideram ativo, o coping no qual há esforços de aproximação (enfrentamento) do individuo ao foco de estresse, enquanto no passivo há evitação ou esquiva.

As escalas de medida desenvolvidas para situações específicas (e.g. coping referido especificamente ao trabalho) são recomendadas por apresentarem estratégias que realmente se aplicam ao contexto em questão, havendo peculiaridades metodológicas a considerar Optou-se, no entanto, por uma medida de coping geral, a Escala de Coping de Billings e MoosBMC (Billings \& Moos, 1981), em versão validada para uso no Brasil (Guimarães, Coêlho \& Freire, 2005) incluindo-se em sua aplicação, estímulos ou episódios e descrições de situações que levavam o respondente a se reportar ao contexto desejado.

\section{Material e Métodos}

Dezoito trabalhadores de todas as áreas operacionais ligadas ao lançamento do foguete VLS-1 que explodiu foram avaliados e, se indicado, tratados. Todos os acometidos (10) por TEPT foram tratados com associação de psicofármacos e psicoterapia. Fezse uso de antidepressivos (sertralina, outros ISRS, venlafaxina, mirtazapina e outros), isoladamente ou em associação com benzodiazepínicos, beta bloqueadores, anticonvulsivantes, incluindo-se os antipsicóticos). A abordagem psicoterápica foi a cognitivo-comportamental (individual e/ou grupal).

Os atendimentos foram feitos semanalmente: medicação, revisão medicamentosa, grupoterapia ( 2 horas) psicoterapia individual (1 hora). Todos os procedimentos eram oferecidos e não obrigatórios. Não houve recusa à participação.

Em uma primeira etapa, os 18 participantes foram avaliados por entrevista clinica e instrumentos de medida. Aqueles que apresentaram diagnóstico de TEPT (10) foram tratados, e em uma segunda etapa, após três meses, reaplicados os mesmos instrumentos. Por motivos operacionais (deslocamentos constantes dos participantes para outras localidades do país e outros países), não foi possível a reavaliação dos demais participantes, todos sem TEPT. Os participantes em tratamento foram monitorados até 12 meses, após a introdução da terapêutica.

Todos os participantes assinaram um Termo de Consentimento Livre e Esclarecido conforme a regulamentação para pesquisa clínica no País e do preconizado pelo CFP. O estudo foi conduzido após a aprovação do Comitê de Ética em Pesquisa da Universidade Católica Dom Bosco (UCDB).

Fez-se análise exploratória de dados (freqüências e porcentagens). A medida de associação utilizada foi a razão de prevalência com intervalo de confiança 
de 95\%. O teste $t$ de Student correlacionou os instrumentos; o teste exato de Fischer comparou as diferenças de resultados entre $1^{\mathrm{a}}$ e $2^{\mathrm{a}}$ avaliações; a regressão linear múltipla verificou se, e em quanto, a variância do TEPT seria explicada pelos sintomas de ansiedade, depressivos ou pelo método de coping. O software estatístico utilizado para as análises foi o XL-STAT.

Os objetivos deste estudo foram: i) caracterizar, antes e após o tratamento psicofarmacológicopsicoterápico, a frequência de Transtorno de Estresse Pós-Traumático (TEPT), sintomas depressivos e ansiosos em trabalhadores do setor aereoespacial de todas das áreas operacionais de lançamento de foguetes; ii) identificar o método de coping utilizado e a relação do primeiro com a resposta ao tratamento.

\section{Instrumentos utilizados}

Avaliou-se o TEPT com o Questionário de Seqüelas do Trauma- QST ( Koverola, Proulx, Hanna, Battle \& Choan, 1992) traduzido, adaptado e validado para o português (Coêlho, 2000). O QST é auto-aplicável e composto de 21 itens que objetivam verificar a ocorrência de TEPT baseado nos critérios do DSM-IV. Para seu preenchimento é necessário que o indivíduo apresente o critério A do DSMIV: vivenciar ou testemunhar um acontecimento extremamente traumático. Como nessa investigação, todos os participantes haviam sido expostos ao mesmo evento traumático, partiu-se do pressuposto que todos preenchiam o critério A. As perguntas 1 a 9 correspondem ao critério $\mathrm{B}$ (re-experienciação intrusiva); perguntas 10 a 16, critério $\mathrm{C}$ (evitação e embotamento) e perguntas 17 a 21 , critério D (excitabilidade aumentada). Esse intrumento pode ser utilizado para diagnóstico e, através de medidas seriadas, também tem por objetico medir a eficácia das intervenções terapêuticas.

Estimou-se a existência de sintomas depressivos e ou ansiosos, dada sua referida comorbidade com o TEPT, apontada na literatura de forma recorrente (Yehuda, 2002; Margis, 2003; Bryant, 2005). Utilizouse uma validação brasileira (Gorenstein \& Andrade, 1996) do Inventário de Depressão de Beck- BDI
(Beck, Ward, Mendelson, Mock \& Erbaugh, 1961). É auto-administrável, contém 21 itens e é utilizado para detectar a presença e o nível dos sintomas depressivos.

A Escala de Ansiedade de Hamilton-HAM-A (Hamilton, 1959) adaptada para uso no Brasil (Sougey, 1987) foi aplicada para avaliação clínica da presença e do nível dos sintomas ansiosos.

Possui 14 itens e cada um oferece cinco opções de resposta, variando de 0 ausência de um determinado sintoma, 1- intensidade ligeira, 2- intensidade forte, 3- intensidade máxima (incapacitante). Sete dos 14 itens estáo relacionados ao humor ansioso, como preocupações excessivas e o restante, a sintomas físicos da ansiedade, tais como desconforto gastrointestinal.

Para avaliação do coping foi utilizada a validação brasileira da Escala de Coping de Billings e MoosBMCP (Amor et al, 2008; Berlim et al, 2003) que avalia o método e o foco de coping usado no enfrentamento de eventos estressantes. É composta por 19 questões dicotômicas (tipo Sim/Não). No presente estudo. somente avaliou-se o método utilizado, se ativo ou passivo (evitação-fuga).

\section{Recursos Humanos e Materiais}

Para as ações empreendidas em campo, foi mantida uma equipe de seis profissionais de saúde mental (três psicólogos e três psiquiatras) durante o período de dois anos. Em algumas etapas, avaliação, e.g., contou-se com mais seis profissionais (três psiquiatras e três psicólogos), treinados especificamente para os objetivos deste estudo. Todo o material gráfico e apoio logístico foi fornecido pela Instituição.

\section{Resultados}

Participaram deste estudo 18 trabalhadores (16 homens; 2 mulheres), todos com cargo de gerente, sendo 16 civis e 2 militares, do Centro Aeroespacial Brasileiro (CTA). Destes, a maioria é composta de: indivíduos brancos $(83,3 \%)$, casados $(88,9 \%)$, com idade entre 46 a 55 anos $(73,2 \%)$ em cargos de coordenação ou chefia $(72,2 \%)$; na função entre 21 a $30 \operatorname{anos}(50 \%)$, de 11 a $20 \operatorname{anos}(33,4 \%)$ e de 5

Tabela 1

Convergência diagnóstica entre QST e entrevista clínica (1 a avaliação)

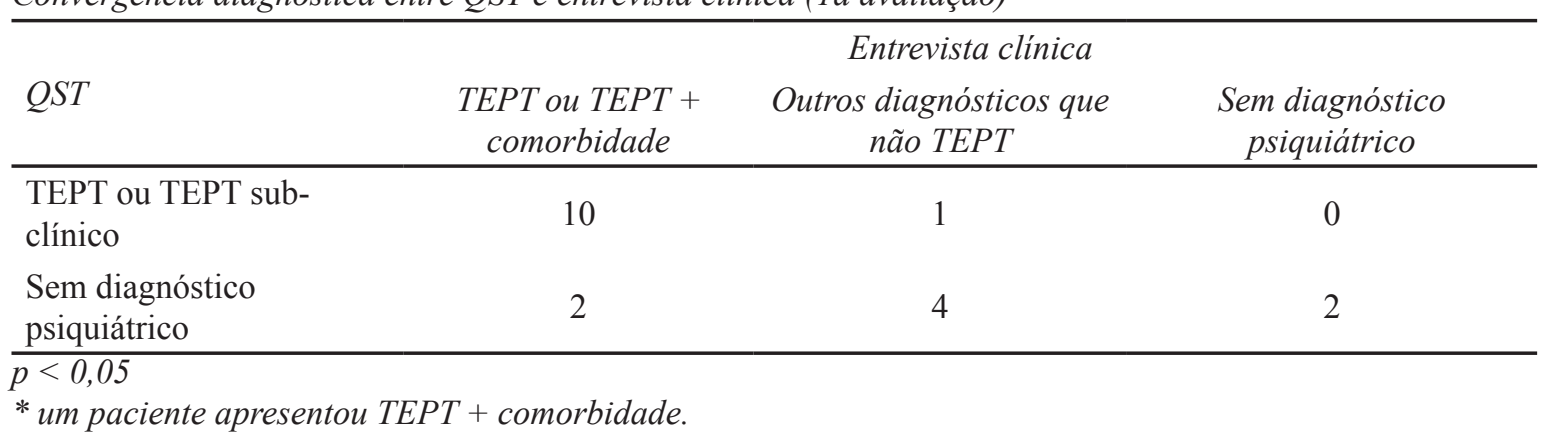


anos até 10 anos (16,7\%); trabalham no CTA entre 21 a 27 anos (44\%), 28 anos ou mais $(27,8 \%)$ e 14 a 20 anos $(27,8 \%)$; presenciaram o acidente de perto, no local do acidente $(38,9 \%)$; presenciaram por vídeo (estavam em São José dos Campos, SP, sede do CTA) (33,3\%); à distância, mas na região do acidente (no Centro de Lançamentos de Alcântara- CLA, MA) $(8 \%)$.

A tabela 1 evidencia que, na primeira avaliação, antes do tratamento psicológico-psiquiátrico, dos 18 participantes, 10 tiveram diagnóstico de TEPT, aferido pelo QST e entrevista clínica, havendo convergência diagnóstica estatisticamente significativa entre as duas ferramentas utilizadas (Teste exato de Fischer: $\mathrm{p}=0,023)$.

Como demonstra a tabela 2, na segunda avaliação pelo QST e entrevista clínica (realizada após três meses de tratamento), encontrou-se diferenças significativas entre as categorias com TEPT e sem TEPT, havendo uma redução de $10(55,5 \%)$, para dois casos $(18,2 \%)$. Houve uma alta convergência diagnóstica entre o QST e a entrevista clínica, com relação à melhora dos resultados (segunda etapa). Dos participantes, 33,3\% (6) apresentavam sintomas ansiosos na primeira avaliação e na segunda, todos, $100 \%$ (10) não mais os apresentavam. Obtiveram-se diferenças significativas entre a primeira e segunda avaliação quanto ao decréscimo dos sintomas de ansiedade (Teste exato de Fischer, $\mathrm{p}=0,02$ )

$\mathrm{Na}$ primeira avaliação, dos 18 participantes, a maioria 55,6\% (10) não apresentou sintomatologia depressiva. Já na segunda, dos 10 avaliados, 90\% (9) não apresentaram sintomatologia depressiva. Observou-se, portanto, decréscimo significativo da sintomatologia depressiva (da primeira para a segunda avaliação). (Teste exato de Fischer: $\mathrm{p}=0,04)$.

Na primeira avaliação, a maioria, 15 (72,3\%) dos participantes apresentou o método ativo de coping (enfrentamento) e $3(27,7 \%)$ o método passivo (evitação/esquiva). Dos 10 participantes da segunda avaliação, 8 (72,3\%) utilizaram o método ativo e 2, evitação/esquiva (dois dos mesmos participantes que apresentaram este método na $1^{\text {a }}$ avaliação). Não foram encontradas diferenças significativas entre a primeira e segunda avaliações com relação ao método de coping utilizado (Teste exato de Fischer: $\mathrm{p}=0$,268).

A tabela 3 mostra diferenças significativas entre melhora ou não da sintomatologia de TEPT e tipo de coping utilizado (teste $t$ de Student: $\mathrm{p}=0,096$ ). As correlações entre os instrumentos para aferir TEPT, ansiedade e depressão, foram baixas e não significativas.

Feita a análise de Regressão Linear Múltipla (tabela 4) a variância do TEPT foi explicada em $68 \%$ pelo tipo de coping utilizado. Participantes com um coping ativo apresentaram uma mais rápida e positiva melhora do quadro de TEPT. Inversamente, participantes com um coping passivo, após o tratamento apresentaram uma resposta mais lenta e negativa.

Tabela 2

Freqüencias e porcentagens do TEPT, sintomas ansiosos, sintomas depressivos e coping, antes ( $1^{a}$ avaliação) e após tratamento psicológico-psiquiátrico ( $2^{a}$ avaliação).

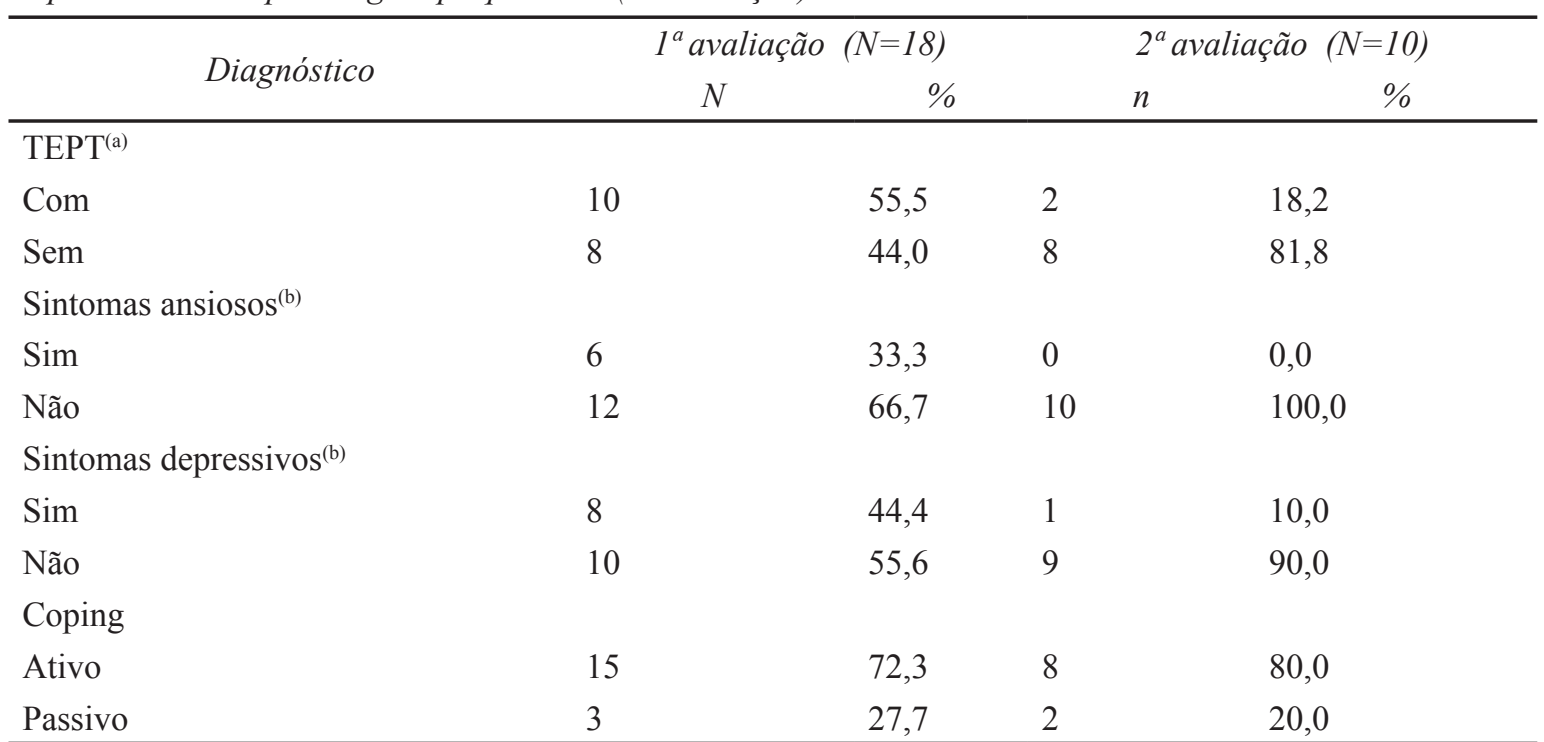

(a) Foram considerados com TEPT, somente os casos com convergência diagnóstica entre o QST e a Entrevista Clínica.

(b) A presença de sintomatologia ansiosa e ou depressiva não implica na presença de diagnóstico nosológico clínico (Transtorno Depressivo e ou Transtorno de Ansiedade). 
Tabela 3

Distribuição das médias, desvios-padrão e correlações entre os instrumentos utilizados

\begin{tabular}{lccc}
\hline \multicolumn{1}{c}{ Instrumentos } & Média $( \pm D P)$ & Correlações & \\
\hline QST (TEPT) & $3,8( \pm 3,7)$ & $\begin{array}{c}\text { TEPTx } \\
\text { ansiedade }\end{array}$ & TEPTx depressão \\
$\begin{array}{l}\text { HAM-A } \\
\text { (Ansiedade) }\end{array}$ & $10,2( \pm 9,7)$ & $* 0,250$ & \\
$\begin{array}{l}\text { BECK } \\
\text { (Depressão) }\end{array}$ & $8,2( \pm 7,3)$ & & $-0,065$ \\
ECBM (Coping) & $59,4 \%( \pm 17,2)$ & & \\
\hline$p<0,05$ & & & \\
\hline
\end{tabular}

\section{Discussão}

O nível de concordância diagnóstica entre o QST e a entrevista clínica, nas duas avaliações foi alto. A possibilidade de haver se realizado um correto diagnóstico, parece ter favorecido uma oferta de terapêuticas adequadas para o enfrentamento do TEPT, o que pode ter concorrido para a redução significativa dos quadros inicialmente apresentados, após três meses de tratamento. As falhas no diagnóstico poderiam ter levado a uma intervenção inadequada, além de agravar os sintomas do TEPT e diminuir a eficácia de futuros tratamentos (Braverman, 1998), o que parece não ter acontecido no presente estudo.

Nessa pesquisa, a sintomatologia ansiosa, bem como a depressiva, não estiveram associadas à presença de TEPT, embora a literatura (Yehuda, 2002; Amor et al, 2008; Berlim et al, 2003) aponte que dentre as patologias comórbidas mais freqüentes se destaquem os transtornos de humor, especialmente a depressão (46\% a 51\%)0 e a ansiedade (29\% a $56,1 \%)$.

Os participantes que apresentavam sintomatologia ansiosa e depressiva na primeira avaliação, após três meses de tratamento tiveram diminuição significativa das mesmas.

Na primeira avaliação realizada observou-se uma frequência de $55.5 \%$ de TEPT (10 participantes de 18). Percentual equivalente ao encontrado na presente investigação corrobora o de outro estudo que apontou uma prevalência de TEPT de 54\%, em vítimas do terrorismo e de tortura (Weisaeth, 1989). Pode-se inferir, portanto, o nível de sofrimento psíquico dos acometidos, mesmo que nem todos foram expostos fisicamente ao acidente.

Na primeira avaliação, dos 10 participantes com TEPT, oito apresentavam um coping ativo e dois um coping passivo. Na segunda avaliação, dos 10 participantes com TEPT (todos tratados), os oito acometidos que apresentaram um coping ativo não mais apresentaram sintomatologia compatível com TEPT e os dois que apresentaram um coping passivo mantiveram quadro de TEPT. A maioria dos participantes apresentou um coping ativo, em concordância com outros autores que encontraram correlação negativa entre a ansiedade e um coping

Tabela 4

Regressão linear múltipla dos fatores da variável preditora TEPT com a variável regressora coping

\begin{tabular}{|c|c|c|}
\hline Variáveis preditoras & $T$ & $\beta$ \\
\hline \multicolumn{3}{|l|}{ TEPT } \\
\hline Sim/Não & $-1,49$ & $-1,58$ \\
\hline \multicolumn{3}{|l|}{ Coping } \\
\hline Ativo & $-0,17$ & $-0,13$ \\
\hline \multirow[t]{4}{*}{ Passivo (evitação-esquiva) } & 0,44 & 0,39 \\
\hline & & $\mathrm{R}^{2}=0,068$ \\
\hline & & $\mathrm{R}^{2}$ ajustado $=0,00$ \\
\hline & & $\mathrm{R}=0,21=0,34$ \\
\hline
\end{tabular}

$p<0,05$ 
pró-ativo (Greenglasss et al, 1999).

Obteve-se associação significativa entre TEPT e coping repercutindo na melhora ou não da sintomatologia, após a introdução do tratamento. Cabe referir que o padrão ou perfil de coping pode ser mais importante do que o uso de qualquer estratégia em particular (Glina et al, 2001). Reconhece-se cada vez mais que as repercussões positivas frente à saúde são produto de um coping eficaz (Antonovsky, 1979).

Algumas limitações no presente estudo: a coleta de dados foi realizada após um mês da ocorrência do evento traumático (a partir do contato feito com a equipe), o que pode explicar a alta frequência de TEPT encontrada. Destaca-se a importância da busca precoce de tratamento psicológico-psiquiátrico, sendo dada ao acometido a oportunidade de organizar suas vivências dolorosas (Kapczinski, 2003).

Não foi possível constituir um grupo controle, dado que, por motivos éticos, todos os acometidos foram tratados, e com a mesma estratégia terapêutica. Portanto, não foram reavaliados os casos diagnosticados sem TEPT (1 ${ }^{\mathrm{a}}$ avaliação), dados os motivos expostos anteriormente.

O reduzido tamanho da amostra $(n=18)$ pode ser considerado uma limitação, lembrando que esta investigação foi conduzida em paralelo à necessidade imperativa de tratamento dos trabalhadores. Procurou-se lidar com o viés de diagnósticoaferição (a equipe saber antecipadamente da alta probabilidade de presença de TEPT), validando-se por meio de entrevista clínica (gold standart) o relato do participante e os resultados obtidos pelo mesmo no QST.

Um aspecto importante em relação à vida dos indivíduos com TEPT é o comprometimento de seu pragmatismo, de sua produtividade, do trabalho, similar ao prejuízo associado às pessoas com depressão, mas menor que o comprometimento dos indivíduos com transtorno do pânico (Glina et al, 2001).

\section{Conclusões}

A frequência de TEPT encontrada foi alta (55,5\%). Os participantes com TEPT e um coping ativo $(80 \%)$ tiveram uma rápida melhora sintomática no pós-tratamento, diferentemente daqueles que apresentavam um coping passivo.

O tipo de coping utilizado parecer ter atuado como moderador para a melhora do TEPT, nessa situação específica. É imprescindível a continuação desta linha de investigações, identificando também, outras variáveis individuais que atuam como fatores de risco ou proteção (internas e externas) para a ocorrência do TEPT, contribuindo-se assim, para o desenvolvimento de ações preventivas e/ou interventivas eficazes.

$\mathrm{O}$ entendimento do TEPT como doença ocupacional é um conceito novo, relevante e fundamental para orientar os serviços de saúde, de administradores e profissionais de recursos humanos na sua prevenção, por meio de treinamento adequado, suporte psicológico-psiquiátrico, emissão da Comunicação de Acidente de Trabalho (CAT) e intervenções na organização do trabalho, visando à melhoria da qualidade de vida geral do trabalhador e da organização.

\section{Referências}

Antonovsky, A. (1979). Health, stress and coping. San Francisco: Jossey-Bass.

Amor, M.J.L., Sánchez, R.J.I., Gancedo, P.J.L., Gonzáles, M.E. (2003 Abr 8) Estrategias terapéuticas en el trastorno por estrés postraumático. Acessado de http://www.interpsiquis.com/2003/ areas/at8/index.html.

Associação Americana de Psiquiatria. (2005) Manual Diagnóstico e Estatístico de Transtornos Mentais-DSM-IV, ( $7^{\mathrm{a}}$ ed.). Porto Alegre: Artes Médicas.

Beck, A. T., Ward, C., H., Mendelson, M., Mock, J. \& Erbaugh, G. (1961) An inventory for measuring depression. Archives of General Psychiatry, 4:561-571.

Billings A, G. \& Moos, R.H. (1981). The role of coping responses and social resources in attenuating the stress of life events. Journal of Behavior Medicine, 4(2):139-157.

Braverman, M. (1998). Posttraumatic stress disorder and its relationship to occupational health and injury prevention. In: J.M Stellman (Ed.) vol I (pp. 33-57): Encyclopaedia of occupational health and safety. 4th ed. Geneva: International Labour Office Press.

Bressan, R.A. et al (2009). The posttraumatic stress disorder project in Brazil: neuropsychological, structural and molecular neuroimaging studies in victims of urban violence. Biomedical Central of Psychiatry. Acessado de http://www.biomedcentral. com $/ 1471.244 \mathrm{x} / 9 / 30$

Bryant, R.A. (2005) Psychosocial approaches of acute stress reactions. CNS Spectr, 10(2):116-122.

Coêlho, A.E.L. (2000) Questionário de seqüelas do trauma: adaptação e tradução para português [apostila]. Campo Grande: UCDB; 2000.

Guimarães, L.A.M.; Coêlho, A.E.L.\& Freire, H.B.G. (2005) Validação da Escala de Coping de Billings e Moos para o português do Brasil [apostila]. Campo Grande: UCDB.

Glina, D.M.; Rocha, L.E.; Batista, M.L.\& Mendonça, M.G. (2001) Mental health and work: a discussion on the connection between work and diagnosis, based on daily practice. Cadernos de Saúde Publica, 17(3):607-616.

Gorenstein, C. \& Andrade, L. (1996). Validation of a Portuguese version of the Beck Depression Inventory and the StateTrait Anxiety Inventory in Brazilian subjects. Brazilian Journal of Biological Medicine, 29(4):453-457.

Greenglass, E., Schwarzer R. \& Taubert T. (1999). The proactive Coping Inventory (PCI): A multidimensional research instrument. Toronto: York University: 1999. Acessado de http:// www.userpage.fberlin.de/health/greenpsi.htm.

Hamilton, M. (1959). The assessment of anxiety states by rating. British Journal of Psychological Medicine, 32(1):50-55.

Kapczinski, F. (2003). Apresentação. Revista Brasileira de Psiquiatria, 25(suppl 1):1-2.

Katz, R..J., Lott, M.H., Arbus, P., Crocq, L., Herlobsen. P., Lingjaerde. O. et al. (1994-1995) Pharmacotherapy of posttraumatic stress disorder with a novel psychotropic. Anxiety, 1(4):169-174.

Kessler, R.C, Sonnega, A., Bromet,. E, Hughes, M. \& Nelson, C.B. (1995). Posttraumatic stress disorder in the national comorbidity survey. Archives of General Psychiatry, 52(12):10481060 .

Koverola, C.; Proulx, J.; Hanna, C., Battle, P. \& Choan, 
M. (1992) Trauma sequelae: A PTSD questionnaire. Manitoba, Canada: University of Manitoba [unpublished manuscript].

Krum, F.M.B.\& Bandeira, D.R. (2009) Enfrentamento de desastres naturais: o uso de um coping coletivo. Paidéia, v. 18, n. 39 pp. $73-84$

Lazarus, R.S. (1966). Psychological stress and the coping process. ( $5^{\text {th }}$ ed). New York: McGraw-Hill.

Margis, R. (2003) Comorbidade no transtorno de estresse póstraumático: regra ou exceção? Revista Brasileira de Psiquiatria 25(suppl 1):17-20.

Negri, L.T., Farci, M.S., Sampaio, A.L.P. \& Guimarães, L.A.M. (2003) Transtorno de estresse pós-traumático relacionado ao trabalho. In: L.A.M.Guimarães \& S. Grubits (Eds), vol. III: Série Saúde Mental e Trabalho (pp.119-130). São Paulo: Casa do Psicólogo.

Rutter, M. (1988). Stress, coping, and development: Some issues and some questions. In: N. Garmez \& M. Rutter (Eds) (pp.141) Stress, coping, and development in children. Baltimore: The Johns Hopkins University Press.

Sougey, E.B (1987). As escalas de avaliação nos ensaios clínicos com benzodiazepínicos: a propósito da escala de ansiedade de Hamilton. Jornal Brasileiro de Psiquiatria, 36:49-53.

Weisaeth, L. (1989). A study of behavioral responses to an industrial disaster. Acta Psychiatrica Scandinavica, 80(suppl 355):13-24.

Yehuda, R. (2002). Post-traumatic stress disorder. New England Journal of Medicine, 346:108.

Recebido: 04/08/2010

Última Revisão: 19/12/2011

Aceite Final: 19/12/2011

Agradecimentos:

Ao CNPq, que por concessão de Bolsa de Produtividade em Pesquisa à primeira autora, possibilitou parte da realização deste estudo (processo 2307306/2004-6).

Aos participantes deste estudo, pela disponibilidade, cooperação e pelo incansável e difícil trabalho no desenvolvimento do setor aeroespacial do Brasil.

Sobre os autores:

Profa. Dra. Liliana Andolpho Magalhães Guimarães - Titular dos Cursos de graduação e de pósgraduação Mestrado em Psicologia da Universidade Católica Dom Bosco- UCDB; Pesquisadora da Universidade de São Paulo- USP- Instituto de Psiquiatria- NUFOR- Setor de Psicologia e Psiquiatria do Trabalho e do CNPq. E-mail: lguimaraes@mpc.com.br.

Dr. Sérgio Nolasco Hora das Neves - Médico-psiquiatra. Mestre e Doutorando pela FCM-UNICAMP; Membro do Laboratório de Saúde Mental e Trabalho do Departamento de Psicologia Médica e Psiquiatria da Faculdade de Ciências Médicas da Universidade Estadual de Campinas (FCM-UNICAMP). E-mail: sergionolasco@hotmail.com. 